\title{
Assessing the Flexibility of Recycled Asphalt Concrete Pavement
}

\author{
Saad Issa Sarsam, Mohammed Chaloob Saleem \\ Department of Civil Engineering, College of Engineering, University of Baghdad, Baghdad, Iraq \\ E-mail: saadisasarsam@coeng.uobaghdad.edu.iq
}

Received: 26 July 2019; Accepted: 24 August 2019; Available online: 30 September 2019

\begin{abstract}
Utilizing reclaimed asphalt pavement (RAP) in new asphalt mixtures has increased in recent years because of its economic and environmental benefits. The flexibility of the recycled asphalt concrete (with cutback and emulsion) in terms of resilient modulus (Mr), rutting resistance, and permanent microstrain have been investigated in this work. Cylindrical specimens of $102 \mathrm{~mm}$ in diameter and $102 \mathrm{~mm}$ in height have been prepared from the recycled mixture after the short-term aging process. Specimens were subjected to 1200 repeated compressive stresses at $(25)^{\circ} \mathrm{C}$. The vertical permanent microstrain was monitored through video capture. It was concluded that RAP mixture can hold the applied loading with minimal permanent deformation as compared to the recycled mixtures. The resilient modulus is lower by (24 and 39) \% for mixes recycled with cutback and emulsion respectively as compared to that of RAP. The rate of strain (slope) increases by $11 \%$ and $4 \%$ when cutback and emulsion were implemented as recycling agent respectively as compared to that for RAP mixture.

Keywords: Recycling; Asphalt concrete; Flexibility; Rutting; Resilient modulus; Permanent strain.
\end{abstract}

\section{Introduction}

The challenge facing road engineers is to develop a sustainable asphalt mixture that reduces the pavement system failure by developing new road materials and new methods of road construction and maintenance [1]. Progress in the road material science has focused on aged and waste materials, such as RAP, likely because of the continued availability of low-cost materials and due to the functional design of asphalt pavements. Recycling can provide the RAP, which is an important economical savings, RAP is usually considered to be a cost-effective pavement construction material which is placed in pavement at increasing percentages [2]. Many researches had indicated the economic benefits of recycling [3]. construction and reconstruction of road pavements imply a considerable consumption of valuable and non-renewable natural resources and the component materials of asphalt mixtures [4]. The properties of the recycled mixture are believed to be mainly influenced by the aged reclaimed asphalt pavement (RAP) binder properties, and the amount of RAP in the mixture [5]. Asphalt binder loses many of its oil components during construction and service resulting in a high proportion of asphaltenes in the blend, which leads to increased stiffness and viscosity of the binder and decreased ductility, making the binder hard and brittle [6]. To recycle this hard and brittle aged pavement, the asphalt must be returned or changed to have the rheological properties of original asphalt. This transformation is completed by adding liquid additives to the mixture being recycled, these additives have been called recycling agents or softening agents [7]. Rejuvenating emulsions are normally used, containing oils that reduce the viscosity of aged asphalt cement, thus improving the adhesion and cohesion properties, as well as the flexibility of the binder. In addition, rejuvenators can penetrate the voids of the pavement, filling them and minimizing the binder oxidation [8]. The rutting resistance of recycled mixtures was studied by [9]. Four mixtures with RAP percentages of $0 \%, 15 \%, 30 \%$ and $50 \%$, were tested. Results obtained from the wheel tracking test indicated that RAP mixtures have very similar rut depth values at the end of the test when calculated between cycles 5000 and 10,000 which means that the presence of RAP in mixtures provides greater resistance to rutting. laboratory investigation of permanent deformation characteristics of asphalt concrete mixes containing reclaimed materials was presented by [10]. The permanent deformation characteristics of asphalt concrete with and without reclaimed materials were evaluated in laboratory using Repeated Load Axial Test and Wheel Tracking Test at a range of test temperatures. Test results showed that the asphalt concrete prepared using reclaimed materials such as waste plastic and Reclaimed Asphalt Pavement (RAP) were more resistance on permanent deformation over a range temperature. The use of resilient modulus testing to compare mixtures compacted with only virgin materials to those compacted with varying amounts of RAP was conducted by [11]. Resilient modulus testing was conducted in accordance with ASTM D 4123-82. The test was performed at 0.33 , 0.5 , and $1 \mathrm{~Hz}$. In a $1-\mathrm{Hz}$ test the applied cycles consisted of a 0.1 -second load followed by a 0.9 -second rest period. 
It was concluded that the resilient modulus rapidly decreases with increasing temperature. This is due to the softening of the asphalt binder as the temperature is increased.

In this investigation, the variation in the flexibility of the RAP and recycled asphalt concrete pavement (with cutback and emulsion) in terms of resilient modulus ( $\mathrm{Mr}$ ), rutting resistance, and permanent microstrain have been investigated.

\section{Materials and methods}

\subsection{Aged materials}

The reclaimed asphalt mixture was obtained by the rubblization of binder course layer of asphalt concrete of the highway in the province of Babylon. This highway was heavily deteriorated with various cracks and ruts existed on the surface. Reclaimed asphalt mixture obtained was assured to be free from deleterious substances and loam that gathered on the top surface. The reclaimed mixture was heated, combined and reduced to testing size as per AASHTO [12]; a representative sample was subjected to Ignition test according to AASHTO T 308, [12] procedure to obtain binder and filler content, gradation and properties of aggregate. Table 1 . Presents the properties of aged materials after Ignition test.

Gradation for the RAP obtained from reclaimed mixture was determined; six samples have been selected randomly from the rubblization process of material stack. These samples were subjected to Ignition test to isolate binder from aggregate and then aggregate was sieved and separated to various sizes to calculate gradation for each sample. The differences between samples were in a minor extent, and the average gradation of the six samples obtained to be the old aggregate gradation is shown in Figure 1 which illustrates that the gradation of old (reclaimed) aggregate for binder layer has slimly deviation with Specification limits of Roads and Bridge SCRB [13].

Table 1. Properties of Aged Materials after Ignition Test

\begin{tabular}{lll}
\hline Material & Property & Value \\
\hline Asphalt binder & Binder content \% & 5.46 \\
Coarse aggregate & Bulk specific gravity & 2.59 \\
& Apparent specific gravity & 2.63 \\
& Wear\% (Los Angeles abrasion) & $23 \%$ \\
Fine aggregate & Bulk specific gravity & 2.601 \\
& Apparent specific gravity & 2.823 \\
Mineral filler & Percent passing sieve no.200 & $98 \%$ \\
& Specific gravity & 2.85 \\
Aged Mixture & Stability kN & 17.4 \\
(Marshall Properties) & Flow mm & 3.05 \\
& Air voids \% & $5.21 \%$ \\
& Bulk density gm/cm & 2.329 \\
& Maximum theoretical density $\mathrm{Gmm} \mathrm{gm} / \mathrm{cm}^{3}$ & 2.465 \\
\hline
\end{tabular}

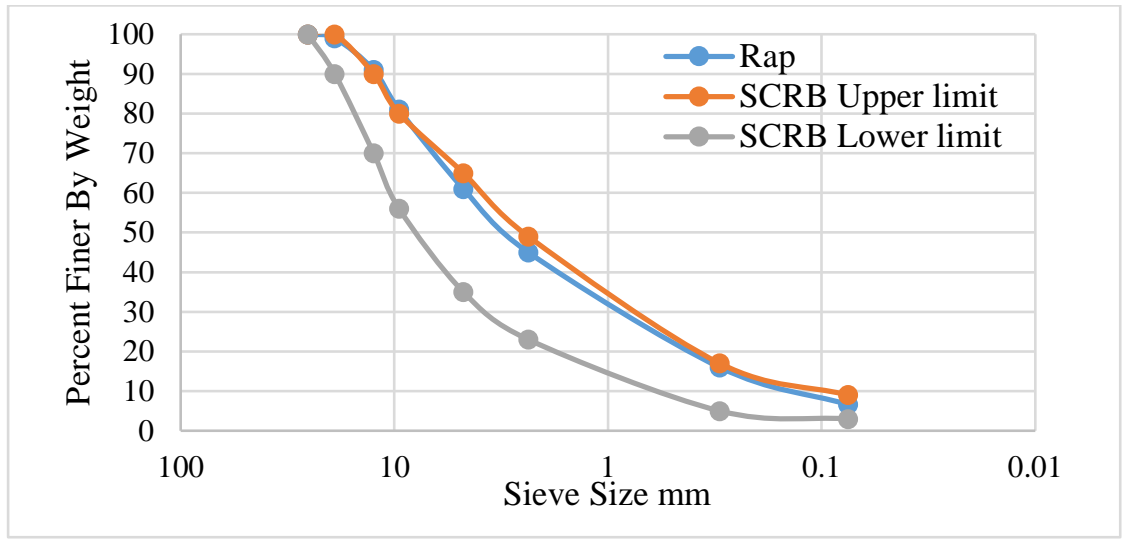

Figure 1. Gradation of RAP (reclaimed) Aggregate Obtained from Aged Mixture 


\subsection{Cutback asphalt}

Medium curing cutback (MC-30) obtained from Al-Dura refinery was adopted for recycling in this work. The properties are listed in Table 2.

Table 2. Properties of medium curing cutback as supplied by the refinery.

\begin{tabular}{|c|c|c|c|}
\hline Property & Test Conditions & ASTM Designation [15] & Value \\
\hline Kinematic viscosity Cst & $60^{\circ} \mathrm{C}$ & D2170 & 42 \\
\hline Flash point ${ }^{\circ} \mathrm{C}$ & - & D3143 & 52 \\
\hline Distillate, volume percent of total distillate & $\begin{array}{l}225^{\circ} \mathrm{C} \\
260^{\circ} \mathrm{C} \\
315^{\circ} \mathrm{C}\end{array}$ & D402 & $\begin{array}{l}23 \\
47 \\
89\end{array}$ \\
\hline Residue from distillation & $360^{\circ} \mathrm{C}$ & D402 & 63 \\
\hline \multicolumn{4}{|c|}{ Percent volume by difference Tests on residue from distillation: } \\
\hline Viscosity Cst & $60^{\circ} \mathrm{C}$ & D2171 & 67 \\
\hline Ductility Cm & $25^{\circ} \mathrm{C}$ & D113 & 132 \\
\hline
\end{tabular}

\subsection{Recycling agents}

Two types of liquid asphalt have been implemented as recycling agent based on available literature [1, 4-6, 14]. They are medium curing cutback, and cationic emulsion.

\subsection{Emulsified asphalt}

Cationic emulsion obtained from ministry of industry and minerals was adopted for recycling in this work, the properties are listed in Table 3.

Table 3. Properties of Cationic emulsion as supplied by the manufacturer

\begin{tabular}{llll}
\hline Property & Test Conditions & ASTM Designation [15] & Value \\
\hline Viscosity Cst & $50^{\circ} \mathrm{C}$ & $\mathrm{D} 7496$ & 235 \\
Storage stability & $24-\mathrm{h}$ & $\mathrm{D} 6930$ & 0.7 \\
Particle Charge & & $\mathrm{D} 7402$ & positive \\
$\begin{array}{l}\text { Distillation: } \\
\text { Oil distillate, by volume of emulsion, \% }\end{array}$ & - & $\mathrm{D} 6997$ & 7 \\
Residue, \% & & 93 \\
\hline Tests on Residue from Distillation & & & \\
Penetration, 25 ${ }^{\circ} \mathrm{C}$ & $25^{\circ} \mathrm{C}, 100 \mathrm{~g}, 5 \mathrm{~S}$ & $\mathrm{D} 5$ & 57 \\
$\begin{array}{l}\text { Ductility Cm } \\
\text { Solubility in trichloroethylene }\end{array}$ & $25^{\circ} \mathrm{C}, 5 \mathrm{~cm} / \mathrm{min}$ & $\mathrm{D} 113$ & 59 \\
\hline
\end{tabular}

\subsection{Recycling of RAP mixture}

Recycled mixture consists of $100 \%$ reclaimed pavement RAP and recycling agent mixed together at specified percentages according to the mixing ratio. First, RAP was heated to approximately $160^{\circ} \mathrm{C}$ and liquid asphalt was added to the heated RAP at the desired amount of $0.5 \%$ by weight of mixture and mixed for two minutes until all mixture was visually coated with recycling agent as addressed by [1]. The recycled mixture was prepared using two types of liquid asphalt: medium curing cutback and cationic emulsion.

\subsection{Preparation of accelerated short term aged recycled mixture}

Recycled mixture was heated to $130^{\circ} \mathrm{C}$ to become loose and then diffuses in shallow trays with $3 \mathrm{~cm}$ thickness and subjected to accelerated aging by laying inside an oven at $135^{\circ} \mathrm{C}$ for 4 hours as per Superpave procedure [12, 16]. The mix was stirred every 30 minutes during the short-term aging to prevent the outside of the mixture from aging more than the inner side because of increased air exposure.

\subsection{Preparation of asphalt concrete specimens}

Cylindrical specimen of $102 \mathrm{~mm}$ in diameter and $102 \mathrm{~mm}$ in height have been prepared from the recycled mixture after the short-term aging process. The mold, spatula, and compaction hammer were heated on a hot plate to a temperature of $150^{\circ} \mathrm{C}$. A piece of non-absorbent paper, cut to size, was placed in the bottom of the mold 
before the mixture was introduced. The asphalt mixture was placed in the preheated mold, and then it was spaded drastically with a heated spatula 15 times around the perimeter and 10 times around the interior. Another piece of non-absorbent paper cut to size was placed on the top of the mix. The temperature of mixture immediately prior to compaction temperature was $150^{\circ} \mathrm{C}$. The mold assembly was placed on the compaction pedestal and subjected to static compaction. The mixture was compressed at top and bottom at temperature $150{ }^{\circ} \mathrm{C}$ under an initial load of $1 \mathrm{Mpa}$ to set the mixture versus the sides of the mold, after that the required load to achieve the target density of $2.372 \mathrm{gm} / \mathrm{cm}^{3}$ was applied for two minutes and the specimen was left to cool at room temperature for 24 hours and then it was removed from the mold using mechanical jack. Specimens were implemented for the Repeated compressive stresses test. Details of obtaining the target density were published elsewhere [17]. Figure 2 exhibit part of the prepared cylindrical specimens.

\subsection{Testing of the specimens under repeated compressive stresses}

Asphalt concrete specimens were subjected to repeated compressive stresses in the pneumatic repeated load system PRLS. The axial repeated load was applied to the specimen and the axial permanent deformation was measured. Compressive loading was applied in the form of rectangular wave with a constant loading frequency of 60 cycles per minute and loading sequence for each cycle is $0.1 \mathrm{sec}$ load duration and $0.9 \mathrm{sec}$. Load repetitions was applied under constant three stress levels of $(0.069,0.138$, and 0.207$) \mathrm{MPa}$, while the testing temperatures of (25) ${ }^{\circ} \mathrm{C}$ was implemented in the test. Figure 3 exhibit the repeated compressive stress setup. The permanent vertical strain is measured as a function of the number of load applications recognizing the fact that the lower permanent strain is related to the lower sensitivity for rutting and corrugation. The accumulation of permanent and resilient strains ( $\varepsilon p$ and $\varepsilon r$ ) were monitored directly through a continuous video capture, while the resilient modulus (Mr) was calculated using equations 1 and 2 [16,18]. Specimens have been tested under three stress levels.

$$
\sigma=\frac{2 p}{\pi t d}
$$

where $\sigma$ is repeated diametral stress $\left(\mathrm{N} / \mathrm{mm}^{2}\right), t$ is thickness of specimen $(\mathrm{mm}), p$ is applied load $(\mathrm{N})$ and $d$ is specimen diameter $(\mathrm{mm})$.

$$
M r=\frac{\sigma}{\varepsilon r}
$$

where $M r$ is resilient modulus $\left(\mathrm{N} / \mathrm{mm}^{2}\right)$ and $\varepsilon r$ is vertical resilient strain $(\mathrm{mm} / \mathrm{mm})$.

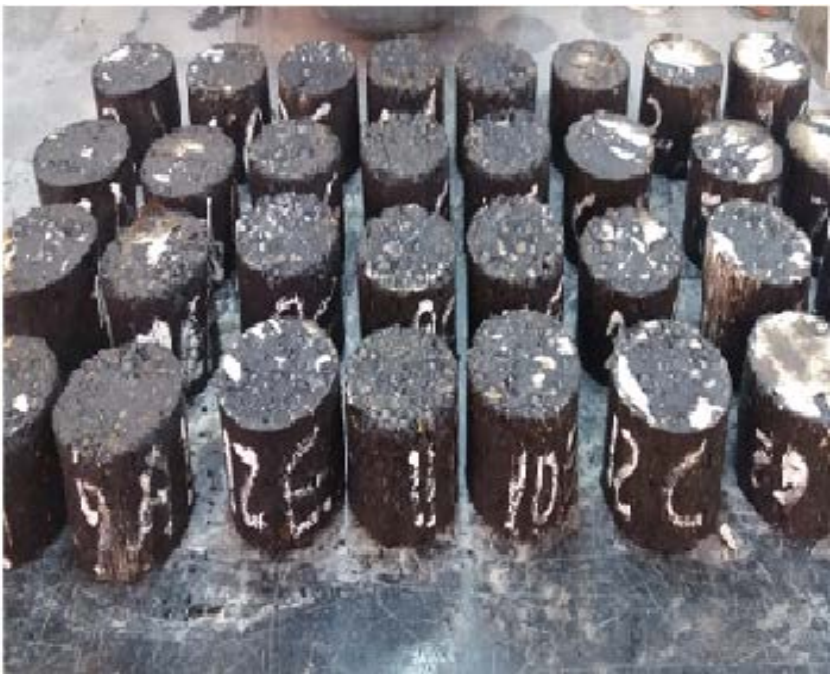

Figure 2. Part of recycled asphalt concrete specimens

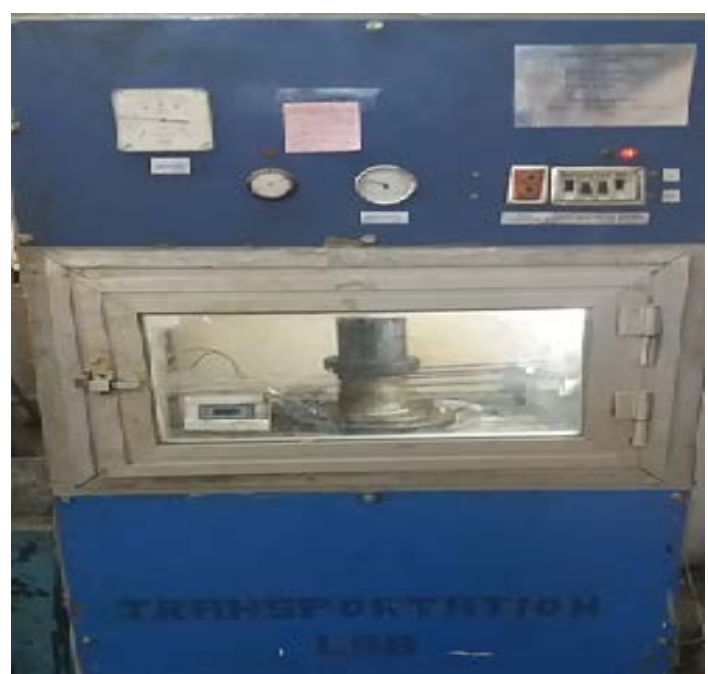

Figure 3. Repeated compressive stress test

\section{Results and discussions}

\subsection{Effect of recycling agent types and stress levels on resilient modulus $(\mathrm{Mr})$}

Figure 4 exhibit the influence of the stresses level on resilient modules for aged and recycled mixture (cutback and emulsion) under compressive stress at $25^{\circ} \mathrm{C}$ after 1200 load repetitions. The resilient modulus increases up to stress level of $0.138 \mathrm{KPa}$, then decreases when the stress level increases to 0.207 $\mathrm{MPa}$. The highest resilient modulus could be achieved at $0.138 \mathrm{MPa}$ level of stress for all mixtures. This may be attributed to that the recycled 
mixture requirement of strength is suitable under the moderate traffic loading condition. Higher stress level of 0.207 MPa will possess extra tensile stresses which the mixture is unable to accommodate, then the resilient modulus is decreased. On the other hand, lower stress level of 0.069 MPa will not exhibit high impact on the resilient modulus. It can also be observed that the RAP mixture exhibits resilient modulus of $171 \mathrm{MPa}$, while the recycled mixtures shows lower resilient modulus by (24 and 39) \% for mixes recycled with cutback and emulsion respectively as compared to that of aged mixture. Such results agree with [16] work.

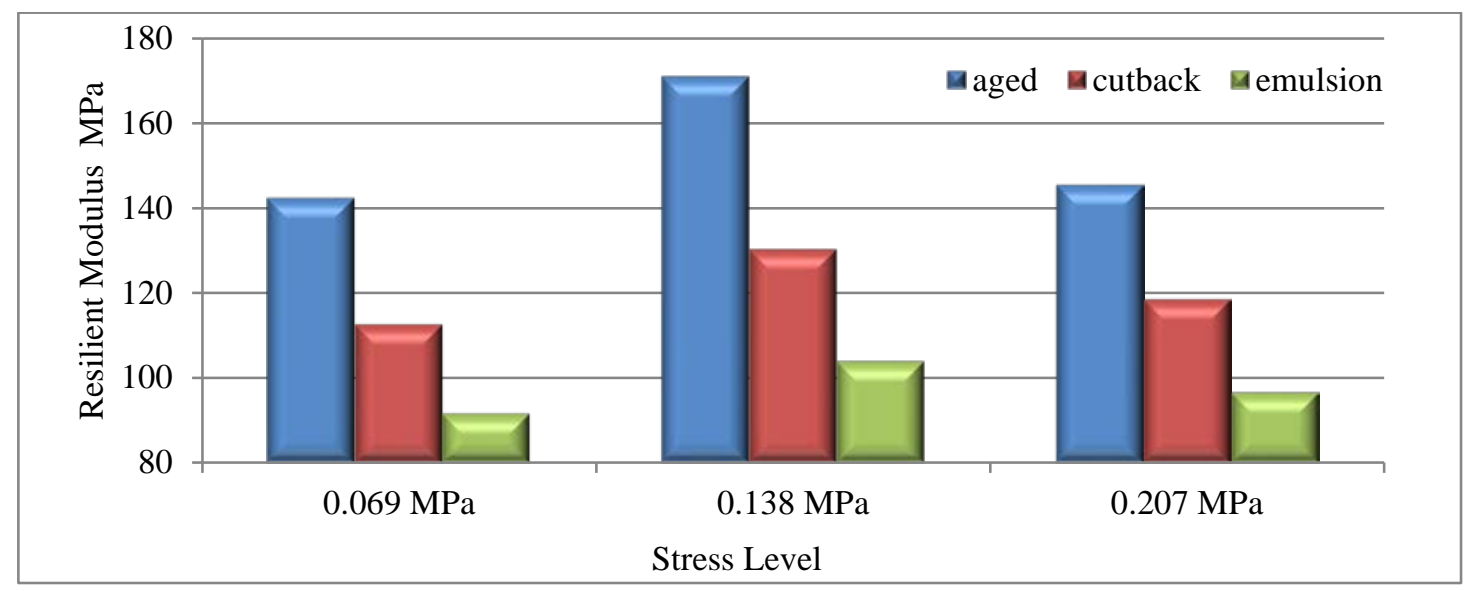

Figure 4. Resilient Modulus (MPa) for various stress levels

\subsection{Effect of recycling agent types and stress level on resistance to permanent deformation}

Figure 5 shows the impact of stresses level on permanent deformation parameter of aged and recycled mixture with (cutback and emulsion) after 1200 load repetitions, it can be observed that while stress level increases, the intercept value and the slope increase as well for different mixtures. The rate of increase for RAP (aged) asphalt concrete is (37.5, and 45.3) \% for 0.138 and $0.207 \mathrm{MPa}$ stress levels as compared to that at $0.069 \mathrm{MPa}$. For recycled mixture with (cutback), the rate of increases is (17, and 25) \% as compared to that at $0.069 \mathrm{MPa}$.

For recycled mixture with (emulsion), the rate of increases is (12, and 17) \% as compared to that at $0.069 \mathrm{MPa}$. The rate of change in slope value is different for different stress level and different mixture. At moderate stress level of $0.138 \mathrm{MPa}$, recycled mixture with cutback asphalt shows lower intercept value by $2 \%$ as compared to that of RAP mixture, while the recycled mixture with emulsion exhibit higher intercept value by $14 \%$ as compared to that of RAP mixture. On the other hand, the rate of strain (slope) increases by $11 \%$ and $4 \%$ when cutback and emulsion were used as recycling agent respectively as compared to that for RAP mixture. this may be attributed to more flexible nature of recycled asphalt concrete as compared to RAP. Such test results agree with $[16,18]$ work.

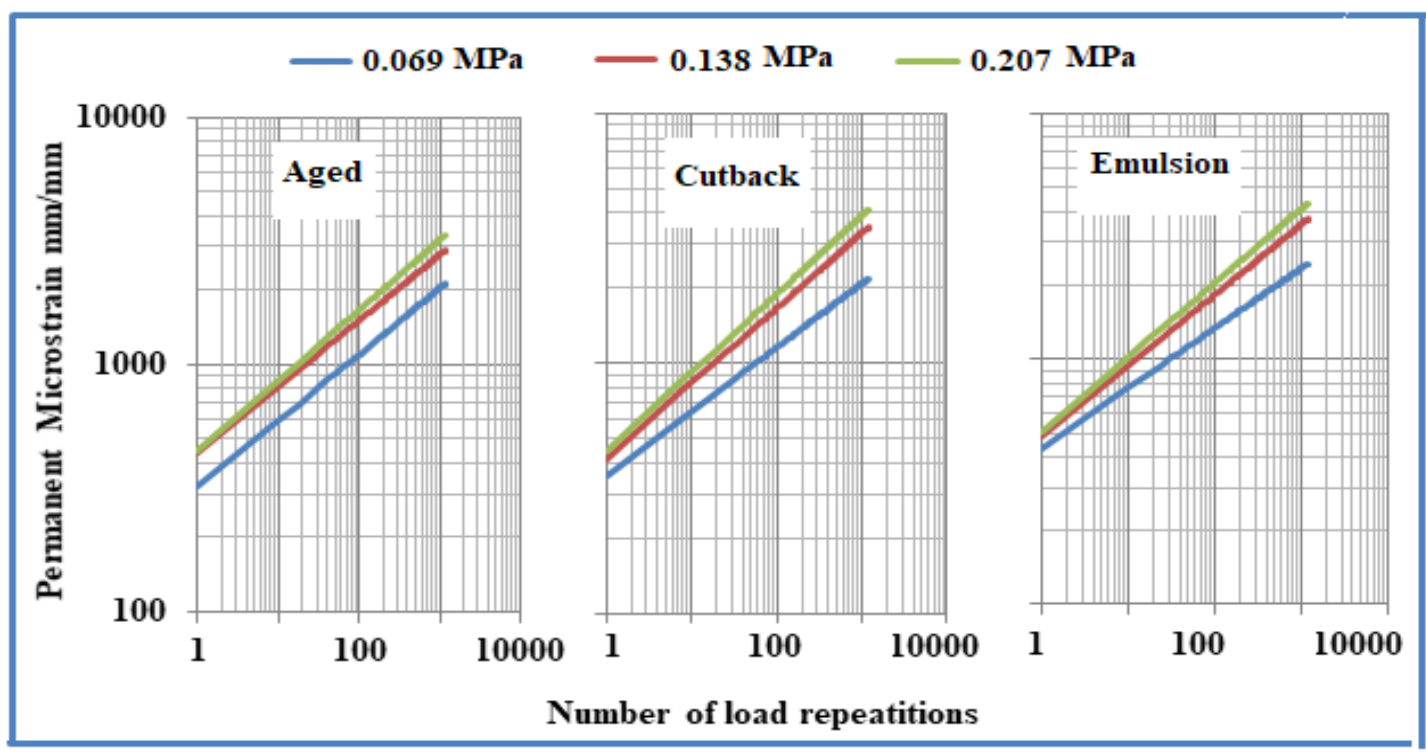

Fig. 5. Typical Relationship Between Permanent Strain and Load Repetition 


\subsection{Resistance to rutting}

Figure 6 exhibit the rutting performance of various asphalt concrete mixtures; it can be observed that the stiffer RAP mixture can hold the applied loading with minimal permanent deformation as compared to the recycled mixtures. At high stress level of $207 \mathrm{kPa}$, the permanent strain increases by (20 and 28) \% for recycled mixtures with cutback and emulsion respectively as compared to RAP mixture. Table 4 demonstrates the mathematical models regarding the resistance to rutting of asphalt concrete where (Y) represent the permanent deformation (microstrain) and (X) denotes the load repetitions. Similar rutting trend was reported by [14].

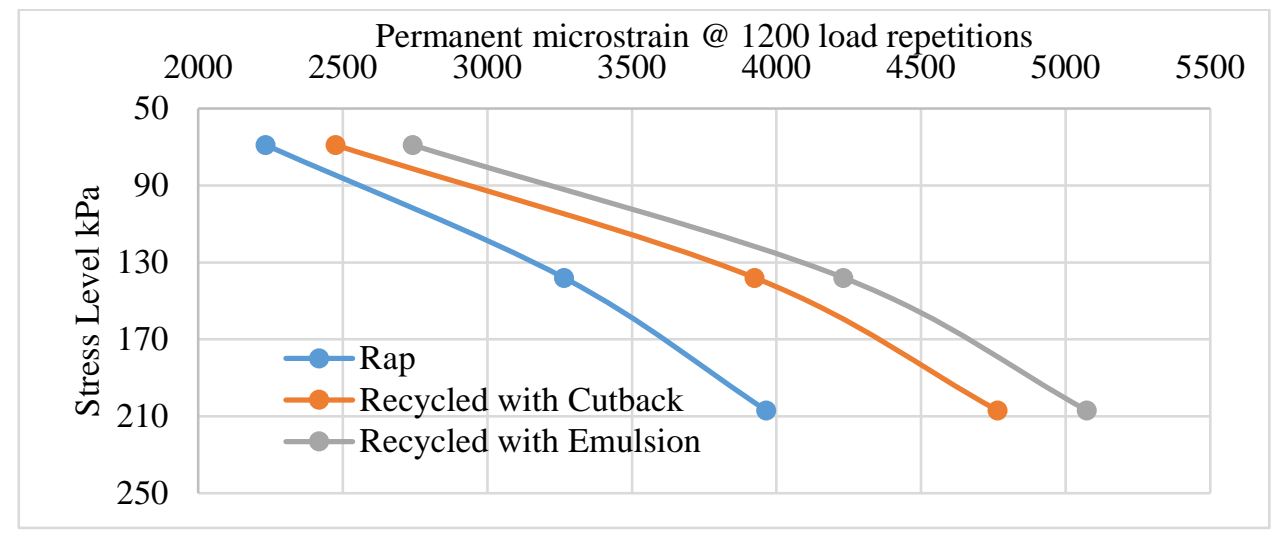

Fig. 6. Rutting Performance of Asphalt Concrete

Table 4. Mathematical Models for Resistance to Rutting at moderate traffic load

\begin{tabular}{lll}
\hline Type of Mixture & Mathematical Model & Coefficient of Determination $\mathrm{R}^{2}$ \\
\hline RAP & $\mathrm{Y}=441.5 \mathrm{X}^{0.2659}$ & 0.953 \\
Recycled with Cutback & $\mathrm{Y}=417.64 \mathrm{X}^{0.3007}$ & 0.944 \\
Recycled with Emulsion & $\mathrm{Y}=486.47 \mathrm{X}^{0.2869}$ & 0.923 \\
\hline
\end{tabular}

\section{Conclusions}

Based on the testing program, the following conclusions could be drawn:

1) The resilient modulus is lower by (24 and 39) \% for mixes recycled with cutback and emulsion respectively as compared to that of RAP (aged) mixture at moderate stress level of $0.138 \mathrm{MPa}$.

2) The resilient modulus increases up to stress level of $0.138 \mathrm{KPa}$, then decreases when the stress level increases to 0.207 MPa. The highest resilient modulus could be achieved at $0.138 \mathrm{MPa}$ level of stress for all mixtures.

3) At moderate stress level of $0.138 \mathrm{MPa}$, recycled mixture with cutback asphalt shows lower intercept value by $2 \%$ as compared to that of RAP mixture, while the recycled mixture with emulsion exhibit higher intercept value by $14 \%$ as compared to that of RAP mixture.

4) The rate of strain (slope) increases by $11 \%$ and $4 \%$ when cutback and emulsion were implemented as recycling agent respectively as compared to that for RAP mixture.

\section{References}

[1] Sarsam SI. A study on aging and recycling of asphalt concrete pavement. University of Sharjah Journal of Pure \& Applied Sciences. 2007;4(2):79-94.

[2] Colbert B, You Z. The determination of mechanical performance of laboratory produced hot mix asphalt mixtures using controlled RAP and virgin aggregate size fractions. Construction and Building Materials. 2012;26(1):655-662.

[3] NCHRP Synthesis 495. Use of reclaimed asphalt pavement and recycled asphalt shingles in asphalt mixtures. A Synthesis of Highway Practice. National Cooperative Highway Research Program. Project 20-05, Topic, 46-05. 2016.

[4] Al-Qadi I, Seifi E, Carpenter S. Reclaimed asphalt pavement-A literature review. Report No. FHWAICT07-001, Illinois Center for Transportation, Rantoul, IL. 2007.

[5] Kandhal PS, Mallick RB. Pavement recycling guidelines for state and local governments participant's reference book. Report No. FHWA-SA-98-042. Federal Highway Administration Office of Engineering. 1997. 
[6] Copeland A. Reclaimed asphalt pavement in asphalt mixtures: state of the practice. Report No. FHWA-HRT11-021. Report Date April 2011.

[7] Silva HM, Oliveira JR, Jesus CM. Are totally recycled hot mix asphalts a sustainable alternative for road paving?. Resources, Conservation and Recycling. 2012;60:38-48.

[8] O’Sullivan KA. Rejuvenation of Reclaimed Asphalt Pavement (RAP) in hot mix asphalt recycling with high RAP content. [M.Sc. thesis]. Worcester Polytechnic Institute. 2011.

[9] Miró R, Valdés G, Martínez A, Segura P, Rodríguez C. Evaluation of high modulus mixture behaviour with high reclaimed asphalt pavement (RAP) percentages for sustainable road construction. Construction and Building Materials. 2011;25(10):3854-3862.

[10] Aschuri I, Woodward D, Woodside A. Permanent deformation characteristics of asphalt concrete containing reclaimed materials. International Society for Maintenance and Rehabilitation of Transportation Infrastructure. technical report. Transportation Research Board. 2009.

[11] Sondag MS, Chadbourn BA, Drescher A. Investigation of Recycled Asphalt Pavement (RAP) mixtures. Report No. MN/RC - 2002-15. Minnesota Department of Transportation. 2002.

[12] AASHTO. Standard specification for transportation materials and methods of sampling and testing. American Association of State Highway and Transportation Officials, 14th Edition, Part II. Washington, D.C.; 2013.

[13] SCRB. General specification for roads and bridges, Section R/9 hot-mix asphalt concrete pavement, Revised Edition. State Corporation of Roads and Bridges, Ministry of Housing and Construction, Republic of Iraq. 2003.

[14] Sarsam SI, AL-Zubaidi I. Resistance to deformation under repeated loading of aged and recycled sustainable pavement. American Journal of Civil and Structural Engineering. 2014;1(2):34-39.

[15] ASTM. Road and paving material, vehicle-pavement system. American Society for Testing and Materials. Annual Book of ASTM Standards; 2009. Vol.04.03.

[16] Sarsam SI, AL-Shujairy A. Influence of recycling agent type on resilient modules and rutting resistance of asphalt concrete pavement. Applied Research Journal, ARJ. 2016;2(1):10-18.

[17] Saleem MC. Influence of liquid asphalt additives on the properties of recycled asphalt concrete. [MSc. Thesis]. College of Engineering, University of Baghdad. 2018.

[18] Sarsam SI, Al-Janabi IA. Assessing shear and compressive strength of reclaimed asphalt concrete. International Journal of Scientific Research in Knowledge. 2014;2(8):352-361.

(c) 2019 by the author(s). This work is licensed under a Creative Commons Attribution 4.0 International License (http://creativecommons.org/licenses/by/4.0/). Authors retain copyright of their work, with first publication rights granted to Tech Reviews Ltd. 